\title{
An interstitial deletion-insertion involving chromosomes 2p25.3 and Xq27.1, near SOX3, causes X-linked recessive hypoparathyroidism
}

\author{
Michael R. Bowl, ${ }^{1}$ M. Andrew Nesbit, ${ }^{1}$ Brian Harding, ${ }^{1}$ Elaine Levy, ${ }^{2}$ \\ Andrew Jefferson, ${ }^{2}$ Emanuela Volpi, ${ }^{2}$ Karine Rizzoti, ${ }^{3}$ Robin Lovell-Badge, ${ }^{3}$ \\ David Schlessinger, ${ }^{4}$ Michael P. Whyte, ${ }^{5,6}$ and Rajesh V. Thakker ${ }^{1}$
}

\begin{abstract}
${ }^{1}$ Academic Endocrine Unit, Nuffield Department of Medicine, University of Oxford, Oxford Centre for Diabetes, Endocrinology and Metabolism (OCDEM), Churchill Hospital, Oxford, United Kingdom. ${ }^{2}$ Molecular Cytogenetics and Microscopy, Wellcome Trust Centre for Human Genetics, University of Oxford, Oxford, United Kingdom. ${ }^{3}$ Division of Developmental Genetics, Medical Research Council (MRC) National Institute for Medical Research, London, United Kingdom. ${ }^{4}$ Laboratory of Genetics, NIH, and National Institute on Aging, Baltimore, Maryland, USA. ${ }^{5}$ Center for Metabolic Bone Disease and Molecular Research, Shriners Hospitals for Children, St. Louis, Missouri, USA. ${ }^{6}$ Division of Bone and Mineral Diseases, Washington University School of Medicine, St. Louis, Missouri, USA.
\end{abstract}

\begin{abstract}
$\mathrm{X}$-linked recessive hypoparathyroidism, due to parathyroid agenesis, has been mapped to a 906-kb region on Xq27 that contains 3 genes ( $A T P 11 C, U 7 s n R N A$, and SOX3), and analyses have not revealed mutations. We therefore characterized this region by combined analysis of single nucleotide polymorphisms and sequencetagged sites. This identified a 23 - to $25-\mathrm{kb}$ deletion, which did not contain genes. However, DNA fiber-FISH and pulsed-field gel electrophoresis revealed an approximately $340-\mathrm{kb}$ insertion that replaced the deleted fragment. Use of flow-sorted $X$ chromosome-specific libraries and DNA sequence analyses revealed that the telomeric and centromeric breakpoints on $X$ were, respectively, approximately $67 \mathrm{~kb}$ downstream of SOX3 and within a repetitive sequence. Use of a monochromosomal somatic cell hybrid panel and metaphase-FISH mapping demonstrated that the insertion originated from $2 \mathrm{p} 25$ and contained a segment of the SNTG2 gene that lacked an open reading frame. However, the deletion-insertion $[\operatorname{del}(X)(q 27.1)$ inv ins $(X ; 2)(q 27.1 ; p 25.3)]$, which represents a novel abnormality causing hypoparathyroidism, could result in a position effect on SOX3 expression. Indeed, $S O X 3$ expression was demonstrated, by in situ hybridization, in the developing parathyroid tissue of mouse embryos between 10.5 and 15.5 days post coitum. Thus, our results indicate a likely new role for SOX3 in the embryonic development of the parathyroid glands.
\end{abstract}

\section{Introduction}

Hypoparathyroidism (HPT) is an endocrine disorder in which hypocalcemia and hyperphosphatemia are the results of a deficiency of parathyroid hormone (PTH) (1). The causes of HPT include trauma to the parathyroids during neck surgery, autoimmune polyendocrinopathy syndrome type 1 , or the complex congential abnormalities of the DiGeorge, Kenny-Caffey, and hypoparathyroidism, deafness, and renal dysplasia (HDR) syndromes $(1,2)$. In addition, HPT may develop as an isolated endocrinopathy that may also be inherited as autosomal dominant (Online Mendelian Inheritance in Man [OMIM] 146200), autosomal recessive (OMIM 146200), or X-linked recessive traits (OMIM 307700) (1, 3-5). Some of the autosomal forms of isolated HPT are due to mutations of the PTH gene $(1,3)$, which is located on chromosome

Nonstandard abbreviations used: ATP11C, adenosine triphosphatase 11C; BAC, bacterial artificial chromosome; BLAST, Basic Local Alignment Search Tool; CASR, calcium-sensing receptor; dpc, days post coitum; HDR, hypoparathyroidism, deafness, and renal dysplasia; HPT, hypoparathyroidism; ISP, insert-specific primer; LOD, $\log _{10}$ of odds; OMIM, Online Mendelian Inheritance in Man; PAC, P1-derived artificial chromosome; PFGE, pulsed-field gel electrophoresis; PH, pleckstrin homology; $\mathrm{PTH}$, parathyroid hormone; RACE, rapid amplification of cDNA ends; $\mathrm{SHH}$, sonic hedgehog; SNP, single nucleotide polymorphism; SNTG2, syntrophin gamma 2; SOX3, Sry-box 3; STS, sequence-tagged site; SU, syntrophin unique; XSP, X chromosome-specific primer; YAC, yeast artificial chromosome.

Conflict of interest: The authors have declared that no conflict of interest exists.

Citation for this article: J. Clin. Invest. 115:2822-2831 (2005).

doi:10.1172/JCI24156.
$11 \mathrm{p} 15$, or the calcium-sensing receptor gene (CASR), which is located on chromosome $3 q 21.3(1,6)$. The gene causing the $\mathrm{X}$-linked recessive form, which seems to involve a developmental gene and has been mapped to Xq27, remains to be identified (7-9).

$\mathrm{X}$-linked recessive HPT (OMIM 307700) has been reported in 2 related multigeneration kindreds from Missouri, USA $(4,5,10)$. Affected subjects, who are males, suffered, if untreated, from neonatal or infantile onset of hypocalcemic seizures $(4,5)$. These individuals have deficiency of circulating immunoreactive PTH, and an autopsy of an affected teenage boy indicated that the HPT was due to parathyroid agenesis (11). Clinical immunodeficiency, facial dysmorphism, deafness, and renal abnormalities, which are notable features of some complex congential anomalies (e.g., DiGeorge, HDR, and Kenny-Caffey syndromes) $(1,12)$, are not present in males with $\mathrm{X}$-linked recessive HPT $(4,5)$. Carrier females, who are the mothers or grandmothers of affected males, give no history of epilepsy and are normocalcemic $(4,5)$. Linkage studies have mapped X-linked recessive HPT to a 906-kb interval (Figure 1) flanked centromerically by a polymorphism associated with the diffuse B cell lymphoma (DBL) proto-oncogene and telomerically by DXS984 in Xq27.1 (7-9). Furthermore, DNA sequence analyses of the 3 genes, adenosine triphosphatase 11C (ATP11C), U7snRNA homologue, and Sry-box 3 (SOX3), that are contained within the $906-\mathrm{kb}$ X-linked recessive HPT critical region have not revealed any abnormalities (9). These findings together with the reported absence of hypocalcemia in a boy with hemophilia B and mental retardation who had a chromosomal deletion that encom- 


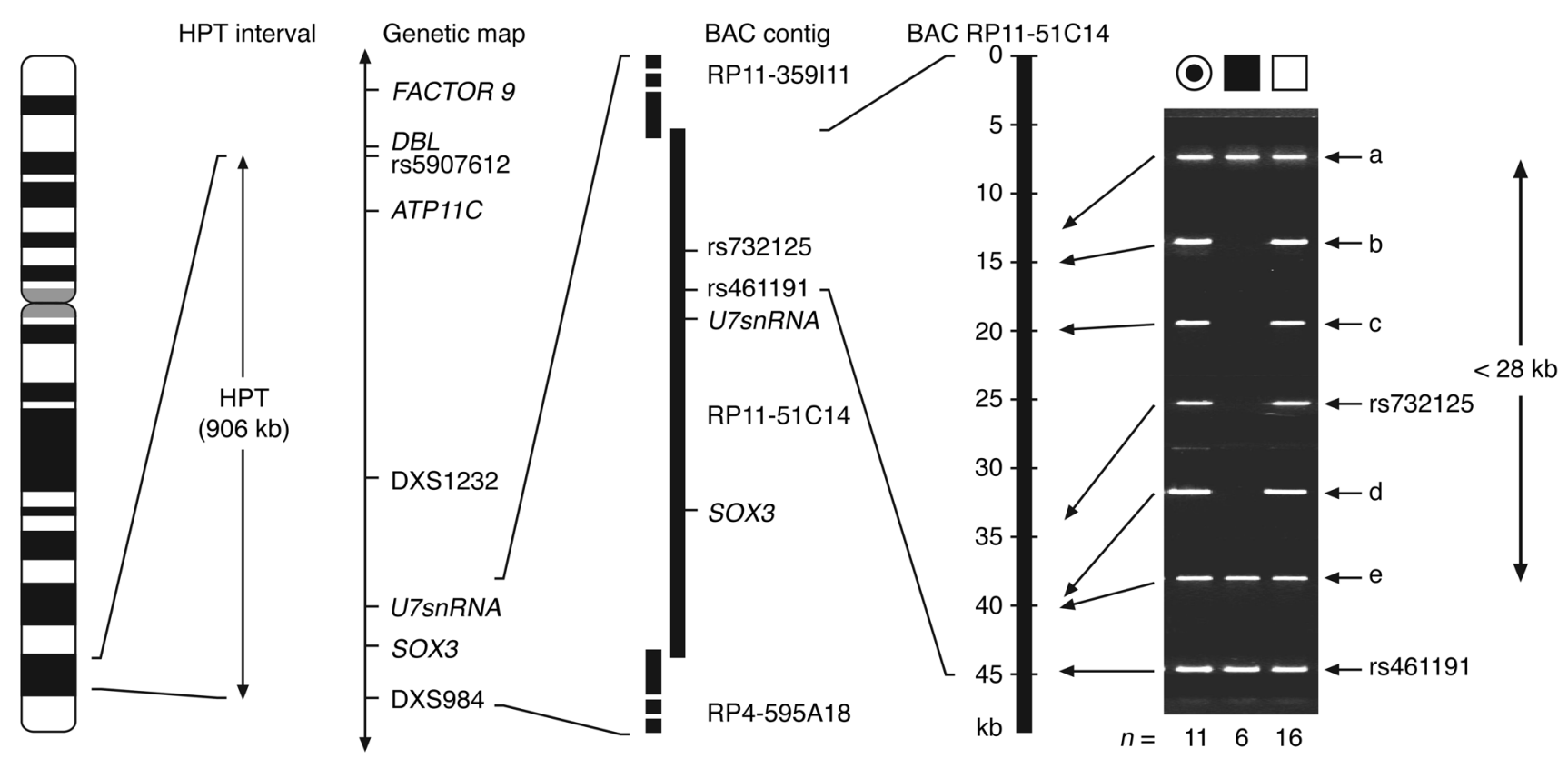

Figure 1

Identification of a less than 28-kb deletion on chromosome Xq27.1 in X-linked recessive HPT patients. The disease locus is located between the SNP rs5907612 and the microsatellite polymorphism DXS984 (9), which are shown on the expanded view alongside the schematically represented X chromosome with Giemsa bands. This 906-kb critical interval contains 3 genes - ATP11C, U7snRNA, and SOX3 — and abnormalities involving the coding regions of these genes in X-linked recessive HPT patients has been previously excluded (9). To further reduce the critical interval, SNPs from the telomeric boundary were initially sought, and analysis of the contig comprising BACs RP11-359l11, RP11-51C14, and RP4-595A18 revealed 2 SNPs, rs732125 and rs461191, that were respectively located approximately $19 \mathrm{~kb}$ and $8 \mathrm{~kb}$ centromeric to U7snRNA. SNP rs461191 proved uninformative in the X-linked recessive HPT kindreds. However, analysis with SNP rs732125 revealed a likely deletion, as PCR products were not obtained from the 6 affected males (filled square) but were obtained from the 16 unaffected males (open square), 11 carrier females (circle with a dot), and 28 unaffected females (data not shown). This deletion was further delineated using 5 STSs (a-e) that were designed at intervals along the sequence of BAC RP11-51C14. PCR products were obtained using STSs a and e but not STSs b, c, and $\mathrm{d}$, thereby indicating that the deletion extended from STS a to STS e, an interval of less than $28 \mathrm{~kb}$.

passed the entire 906-kb interval containing ATP11C, U7snRNA, and SOX3 genes $(8,9,13)$ suggest that other genomic abnormalities such as duplications or translocations, which could cause altered gene function (14-19), may underlie the etiology of X-linked recessive HPT. We therefore undertook a detailed characterization of the interval containing the X-linked recessive HPT locus.

\section{Results}

Identification of a deletion-insertion in X-linked recessive HPT. Our initial characterization of the X-linked recessive HPT interval began with the identification of single nucleotide polymorphisms (SNPs) for segregation studies, with a view to reducing the critical region by identifying recombinants between the disease and SNP loci. The interval between the DBL-associated SNP, rs5907612, and DXS984 (Figure 1), which is $906 \mathrm{~kb}$ in size (9), is contained within a contig formed by 12 bacterial artificial chromosomes (BACs), and a search using the GenBank database revealed 2 SNPs (rs732125 and rs461191) centromeric to the loci for U7snRNA and SOX3. The family members of the $2 \mathrm{X}$-linked recessive HPT kindreds, $\mathrm{P} / 60$ and $\mathrm{W} / 81(4,5)$, were homozygous and hence uninformative for SNP rs461191. However, a deletion involving SNP rs732125 was detected in the 6 males affected with X-linked recessive HPT, and use of 5 sequence-tagged sites (STSs) derived from the DNA sequence of BAC RP11-51C14 revealed that this deletion was less than or equal to $28 \mathrm{~kb}$ in size (Figure 1).
The use of long-range SfiI restriction endonuclease mapping utilizing pulsed-field gel electrophoresis (PFGE) studies (20) with a $\left[{ }^{32} \mathrm{P}\right]$-labeled SOX3 probe (Figure 2) and long-range PCR (data not shown) to confirm this deletion did not reveal the expected loss of a DNA fragment up to $28 \mathrm{~kb}$ in size. Instead, a gain of a large insertion at the site of the deletion was observed (Figure 2). In order to characterize the boundaries of this deletion-insertion and to determine its DNA sequence, vectorette libraries (20-22) of genomic DNA digested with BamHI, EcoRI, HindIII, HpaII, and RsaI were constructed for both a normal male (individual IV.14; Figure 2) and a male affected with X-linked recessive HPT (individual IV.15). In addition, X chromosome-specific EcoRI, RsaI, and TaqI vectorette libraries were constructed using flow-sorted chromosomes from a male affected with X-linked recessive HPT (individual IV.15; Figure 2). PCR products were generated utilizing $X$ chromosome-specific and vectorette unit binding primers, and amplicons containing the breakpoints were identified by use of an oligomer-extension "hot blot" assay (23). These targeted PCR products were subcloned into pGEM-T and their DNA sequences determined (24). This revealed that the telomeric breakpoint had occurred between nucleotides 38,597 and 38,598 of BAC RP11-51C14 (Figure 1). However, the centromeric breakpoint had occurred within a $2 \mathrm{~kb}$ (at) dinucleotide-rich repetitive sequence. This rendered it difficult to establish the exact position of the centromeric breakpoint within this region, which spanned between nucleotides 13,500 and 15,500 of 

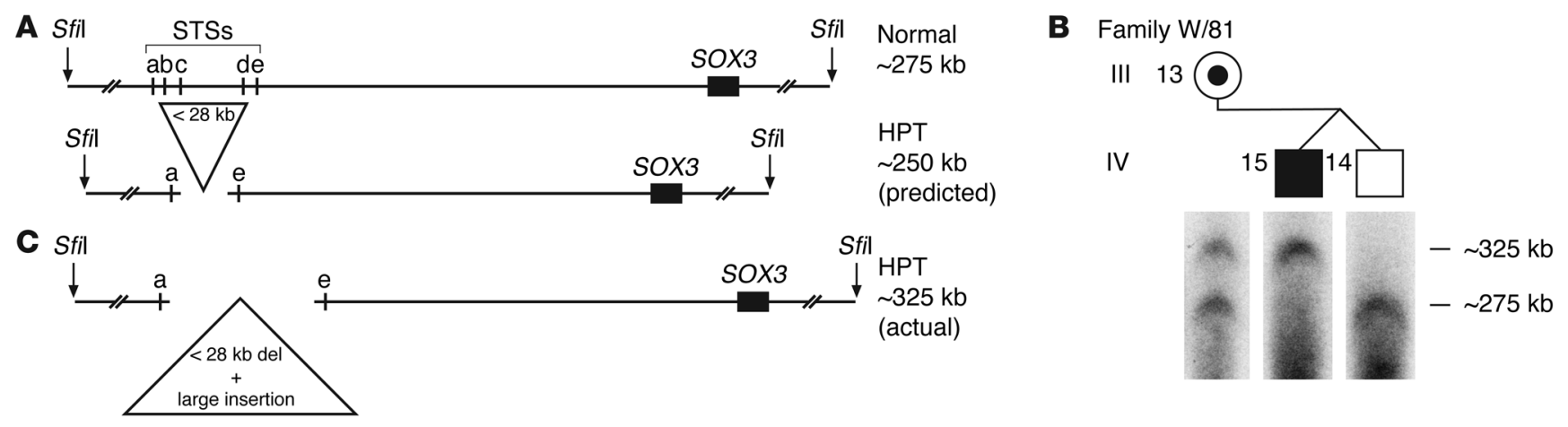

Figure 2

Identification of a large insertion on chromosome Xq27.1 in X-linked recessive HPT patients. In order to confirm the less than 28-kb deletion involving STSs b, c, and d and SNP rs732125 (Figure 1) in X-linked recessive HPT patients, PFGE restriction mapping studies (20) were undertaken. (A) The less than 28-kb deletion predicted the occurrence of an approximately 250-kb Sfil fragment in X-linked recessive HPT patients, as compared with an approximately 275-kb Sfil fragment in normal individuals, when a [32P]-labeled SOX3-derived probe for hybridization was used. (B) PFGE confirmed the presence of an approximately $275-\mathrm{kb}$ Sfil fragment in normal males (open square) but revealed an unexpected larger Sfil fragment of approximately $325 \mathrm{~kb}$ in males affected with X-linked recessive HPT (filled square). Carrier females (circle with a dot) had both the approximately $275-\mathrm{kb}$ and $325-\mathrm{kb}$ Sfil fragments. The results from 3 members of family W/81 $(5,8)$, which consists of 48 members, are shown, and the individual's identification code regarding generation number and position in the pedigree is as previously reported (8) to facilitate comparison between the studies. (C) These findings are consistent with the occurrence of a large, at least 75-kb [i.e., (325-275) $+28 \mathrm{~kb}$ ] insertion at the site of the less than 28 -kb deletion (del) in individuals affected with X-linked recessive HPT.

BAC RP11-51C14 (Figure 1). These findings established that the deletion was between 23 and $25 \mathrm{~kb}$ in size and involved Xq27.1. An approximately 1,750-bp Bam HI vectorette PCR product encompassing the telomeric breakpoint, and adjoining insert DNA was sequenced. A Basic Local Alignment Search Tool (BLAST) analysis, using the versions available in 2001-2003, of this insert sequence obtained from the telomeric breakpoint did not identify any matches in the databases. This sequence was used to design insertspecific primer 1 (ISP1) and ISP2 that would generate insert-specific PCR products (Figure 3). These were used with DNA from a monochromosomal rodent-human somatic cell hybrid panel (25), and the insert-specific sequence was shown to originate from human chromosome 2 (Figure 3 ). In addition, a third ISP, ISP3, was used in conjunction with $\underline{X}$ chromosome-specific primer $\mathrm{R}$ (XSPR) to generate a hybrid PCR product that was specific for the HPT telomeric boundary (Figure 3). This revealed the hybrid PCR product, which was $321 \mathrm{bp}$ in size (Figure 3 ), to be present in all of the 6 hemizygous males affected with HPT and in all of the 11 carrier females, who were heterozygous and also had the normal $237-b p$ product. The hybrid PCR product was not present in any of the 16 unaffected males or 28 unaffected females, all of whom had the normal PCR product. Thus, there was cosegregation, with a peak $\log _{10}$ of odds (LOD) score of 8.10 at $0 \%$ recombination, of the deletion-insertion with HPT in the 2 related kindreds.

Characterization of the insertion reveals origin from chromosome $2 p 25.3$. The ISPs (ISP1 and ISP2) were utilized to generate a 1,181-bp PCR product (Figure 3) that was radiolabeled and used to screen chromosome 2-specific P1-derived artificial chromosome (PAC) (LL02NP04) and Fosmid (LL02NC03) libraries (26) and the Centre d'Études du Polymorphisme Humain (CEPH) megaYAC library (27). This yielded 1 positive fosmid clone, AG63A8, and 1 nonchimeric yeast artificial chromosome (YAC) clone, 972C12. Combined use of the fosmid clone, from chromosome 2, with BAC RP11-51C14, from Xq27, in dual-color FISH analyses (28) of metaphase spreads, confirmed the presence of the insertion at Xq27.1 and revealed that it originated from chromosome 2p25 (Figure 4). Thus, in normal males hybridization with fosmid AG63A8 yielded signals from chromosome 2 pter only; whereas, in males affected with X-linked recessive HPT and in female carriers, fosmid AG63A8 hybridization signals were observed from chromosome 2 pter and Xqter. Furthermore, the merging of the green signal, from fosmid AG63A8, with the red signal, from BAC RP11-51C14, on the $\mathrm{X}$ chromosome harboring the deletion-insertion to yield a yellow signal and the use of DNA fibre-FISH analysis (28) revealed that the 2 p25 insertion was adjacent to the BAC RP11-51C14 sequence (Figure 4). Thus, a contiguous signal was obtained from the $2 \mathrm{X}$ chromosome BACs RP11-359I11 and RP11-51C14 in the normal male, consistent with their juxtaposed locations (Figures 1 and 4). However, such a contiguous signal was not observed in the male affected with X-linked recessive HPT, whereas use of the chromosome 2 p25-derived YAC $972 \mathrm{C} 12$ yielded a contiguous signal (Figure 4). These findings, which indicate the insertion of chromosome $2 \mathrm{p} 25$ sequence within that of $\mathrm{Xq} 27.1$ sequence, are consistent with the presence of a deletion-insertion in the male affected with X-linked recessive HPT. This event is likely to have arisen in the founder, more than 6 generations ago $(4,5)$, by nonhomologous recombination that probably involved the (at) dinucleotide-rich repetitive sequence at the centromeric breakpoint of the deletion on Xq27.1 (Figure 3). The precise nature of any other associated chromosomal abnormalities, such as deletions of $2 \mathrm{p} 25$, in the founder cannot be determined. However, it is important to note that males affected with X-linked recessive HPT and carrier females have normal copies of chromosome 2 (Figure 4). Thus, the occurrence of X-linked recessive HPT in the progeny of the founder is due to inheritance of the abnormal $\mathrm{X}$ chromosome that harbors the deletion-insertion involving chromosomes Xq27.1 and 2p25.

The extent of the chromosome $2 \mathrm{p} 25$ insert was further characterized by first determining the end sequences (20) of YAC $972 \mathrm{C} 12$ and then identifying BACs that contained these sequences, with the aim of assembling a tiled path of such clones (Figure 4). BACs 

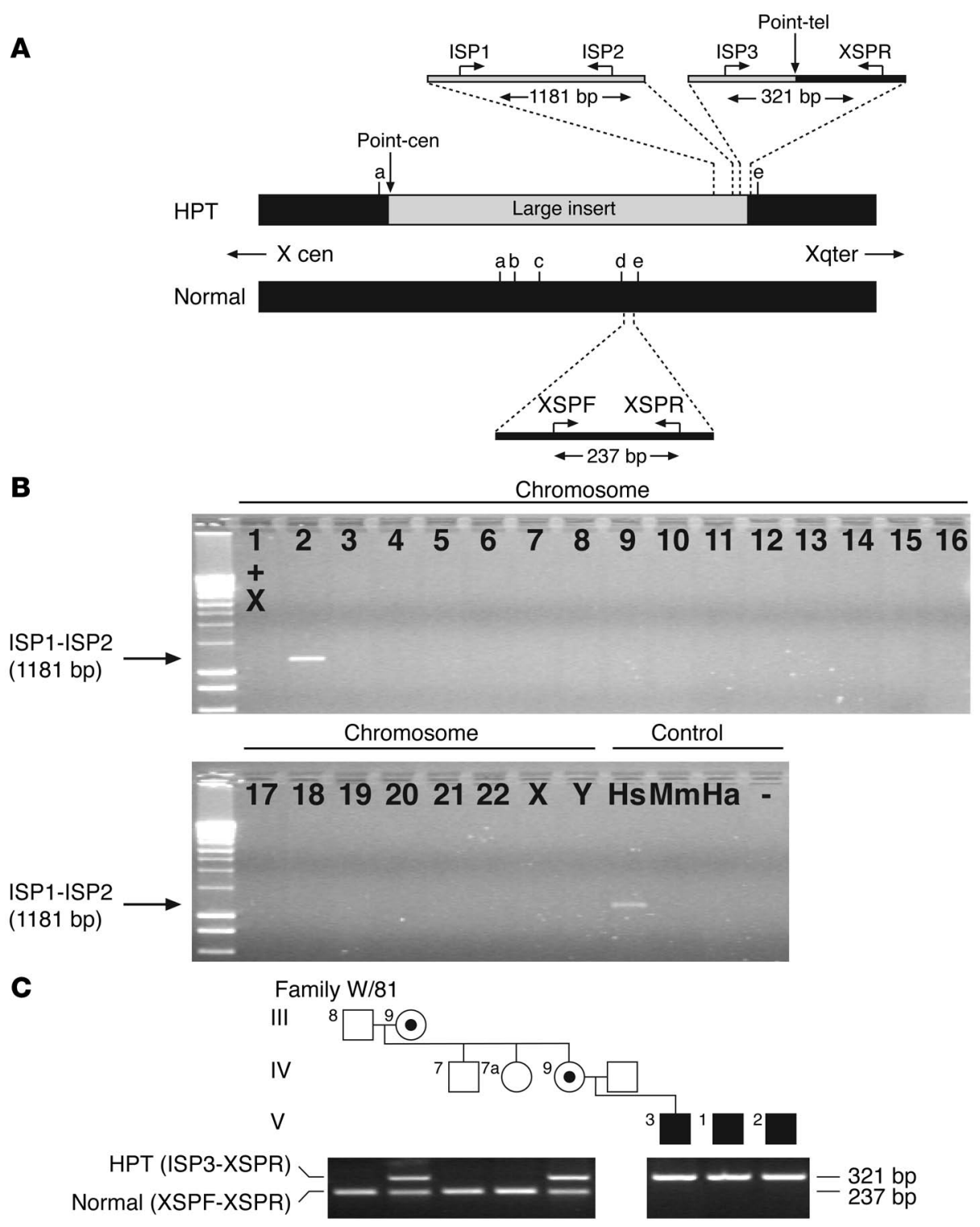

Figure 3

Characterization of the deletion-insertion in X-linked recessive HPT. (A) The DNA sequences of the centromeric and telomeric breakpoints (Point-cen and Point-tel, respectively) were determined using genomic libraries generated from EBV-lymphoblastoid DNA and flow-sorted $X$ chromosomes of an affected male (22). The DNA sequence from the centromeric boundary consisted of a dinucleotide repeat, and the DNA sequence from the telomeric boundary was therefore used to design ISPs (ISP1, ISP2, and ISP3) and XSPS (XSPF and XSPR) for the further characterization of the insertion. The sizes of the PCR products obtained with each primer pair are indicated. The locations of STSs a-e (Figure 1) are shown. (B) Use of primers ISP1 and ISP2 and DNA from a monochromosomal somatic cell hybrid panel revealed that the insert sequence originated from chromosome 2. 1+X, DNA from cell with chromosomes 1 and $\mathrm{X}$; Hs, human DNA; Mm, mouse DNA; $\mathrm{Ha}$, hamster DNA; -, no DNA. (C) Use of primers ISP 3 and XSPR, which flank the telomeric breakpoint, identified the deletion-insertion in affected males (filled squares) and carrier females (circles with a dot). Use of primers XSPF and $X S P R$ identified the normal $X$ chromosome allele in unaffected males (open squares), unaffected females (open circles), and carrier females. The results from 8 members of family W/81 are shown, and the identification code used for individuals is as described in Figure 2. Individual $7 \mathrm{a}$, who is an unaffected sister, has not been reported in previous studies (8). The combined results from all the members of families W/81(8) and $\mathrm{P} / 60$ (8) yielded a LOD score of 8.1 at $0 \%$ recombination.
RP11-455F15 and RP13-542C4 contained DNA sequences that matched the left- and right-arm end sequences of YAC 972C12, respectively, and the sequences of these BACs were in turn used to identify 6 other BACs (i.e., RP13-539J13, RP11-97B21, CTD2029L3, RP11-1268F2, RP13-932E12, and RP11-1294H20) and 1 fosmid (XXfos-83269E11) to assemble a contig. The size of the insert sequence was estimated to be less than or equal to $500 \mathrm{~kb}$ by DNA fiber-FISH analysis (28) that utilized the chromosome Xq27 BACs RP11-359I11 and RP11-51C14 and the chromosome 2p25-derived YAC $972 \mathrm{C} 12$ (Figure 4). These results also revealed that the entire $2 \mathrm{p} 25$ insert was contained within the $750-\mathrm{kb}$ sequence of YAC $972 \mathrm{C} 12$. The use of DNA from these clones and ISPs (ISP1 and ISP2; Figure 3) for the telomeric boundary in PCRs established that this breakpoint was on BAC CTD-2029L3, and the use of metaphase-FISH analysis (28) confirmed this and revealed that the entire $176-\mathrm{kb}$ sequence of RP11-1268F2 was contained within the insert and that RP13-542C4 harbored the centromeric boundary (Figure 4). These results also revealed that the chromosome
2 p25 sequence was inverted upon insertion at Xq27.1 (Figure 5). DNA sequence analysis of the chromosome 2p25 clones and the insert revealed that the $2 \mathrm{p}$ telomeric breakpoint of the insert was between nucleotides 94,427 and 94,428 of BAC CTD-2029L3 and that the centromeric breakpoint was located between nucleotides 40,000 and 75,000 of BAC RP13-542C4, thereby indicating that the size of the insert was $305-340 \mathrm{~kb}$ and from $2 \mathrm{p} 25.3$. Thus, $\mathrm{X}$-linked recessive HPT is caused by a molecular deletion-insertion $[\operatorname{del}(X)(q 27.1)$ inv ins $(X ; 2)(q 27.1 ; \mathrm{p} 25.3)]$ involving a loss of $23-25 \mathrm{~kb}$ from Xq27.1 and an inverted insertion of $305-340 \mathrm{~kb}$ from 2 p25.3 to Xq27.1. A search for such a deletion-insertion and other deletions or insertions in 9 unrelated males with idiopathic HPT using PCR primers for the 2 SNPs (rs732125 and rs461191; Figure 1), the 5 STSs (a-e; Figure 1), and the telomeric breakpoint of X-linked recessive HPT (ISP3 and XSPR; Figure 3) did not reveal any abnormalities, thereby indicating that the deletion-insertion $\operatorname{del}(\mathrm{X})(\mathrm{q} 27.1)$ inv ins $(\mathrm{X} ; 2)(\mathrm{q} 27.1 ; \mathrm{p} 25.3)$ is likely to be a rare event that has occurred in the 2 related kindreds from Missouri (8). 
A

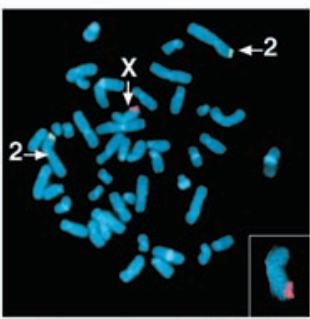

Normal male (IV.14)

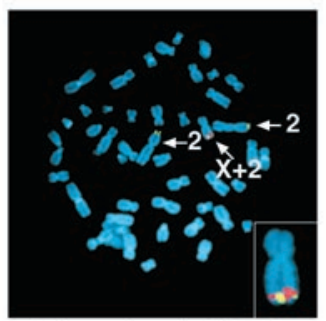

Affected male (IV.15)

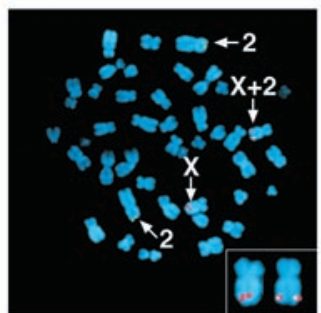

Carrier female (III.13)
B

\section{Normal male (IV.14)}

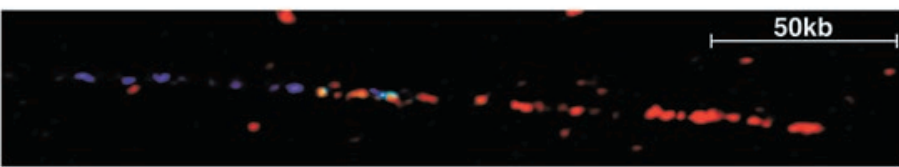
$n>20$

\section{Affected male (IV.15)}

C

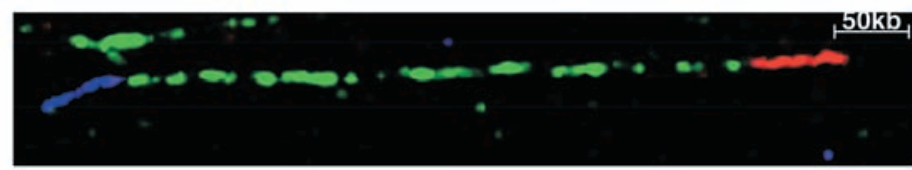

Left arm

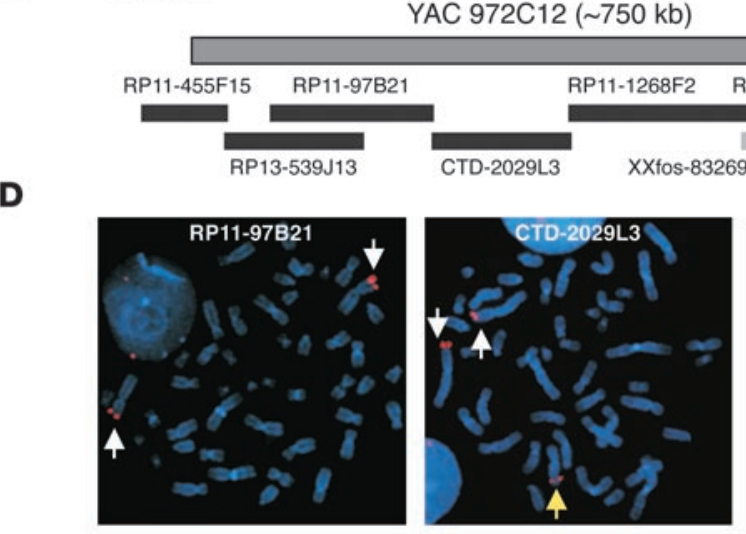

YAC $972 C 12(\sim 750 \mathrm{~kb})$

Right arm

Affected male (IV.15)

\section{Figure 4}

FISH analyses confirm the deletion-insertion involving Xq27.1 and 2p25 in X-linked recessive HPT. (A) Metaphase-FISH analysis, using 2p25 fosmid AG63A8 (green signal) and Xq27 BAC RP11-51C14 (red signal). Chromosomes counterstained with DAPI (blue), and arrows indicate chromosomes 2 and $\mathrm{X}$, as well as and abnormal $X$ containing insert from 2p25 $(X+2)$. Insets show enlarged views of $X$ chromosome. Juxtaposed signals of fosmid and BAC, at Xq27, due to the deletion-insertion, yielded the yellow color. (B) Use of 3-color DNA fiber-FISH analyses revealed a large insertion that was derived from 2p25, at Xq27 in affected males. DNA fibers were hybridized with 2p25 YAC $972 \mathrm{C} 12$ (size, $750 \mathrm{~kb}$ ), and the Xq27 BACs (Figure 1) RP11-51C14 (size, $149 \mathrm{~kb}$ ) and RP11-359l11 (size, $43 \mathrm{~kb}$ ). YAC $972 \mathrm{C} 12$ was labeled with fluorescein, BAC RP11-51C14 with Alexa-594, and BAC RP11-359I11 with Cy5, to yield green, red, and far red (pseudocolored in blue) signals, respectively. The numbers of observed DNA fibers yielding signals are indicated. (C) Contig of BACs (solid bars) and fosmid (broken bar) for YAC 972C12. (D) BACs were labeled with Texas red and separately hybridized to metaphase chromosome spreads. Two red signals corresponding to 2p25 hybridizations (white arrows) were obtained for each BAC (data shown for BACs RP11-97B21, CTD-2029L3, and RP13$542 \mathrm{C} 4$ ), and a third signal corresponding to Xq27 hybridizations (yellow arrows) was also detected for BACs CTD-2029L3, RP11-1268F2 (data not shown), and RP13-542C4. These results indicate that the insertion at Xq27.1 is contained between CTD-2029L3 and RP13$542 \mathrm{C} 4$ and is approximately $305-340 \mathrm{~kb}$ in size. The identification code used for individuals is as described in Figure 2.
Search for expressed sequences within the deletion-insertion. In order to elucidate further the genetic consequences of this complex chromosomal rearrangement associated with $\mathrm{X}$-linked recessive HPT, we investigated the involved DNA sequences for expressed genes. The 2 p25.3 inserted $305-340 \mathrm{~kb}$ sequence contained a large segment of the syntrophin gamma 2 (SNTG2; NM_018968) gene, that consists of the $3^{\prime}$ part of intron 1 together with exons 2 to 16 and the $5^{\prime}$ part of intron 16 . Thus, this inserted segment of the SNTG2 gene did not contain the normally utilized start and stop codons, which are in exons 1 and 17, respectively (29). However, SNTG2 has been reported to have alternative transcripts (29), and our analysis of the DNA sequences indicated that there may be alternative exons within the 2 p25.3 inserted approximately $61 \mathrm{~kb}$ of intron 1 and the adjacent Xq27.1 sequence (Figure 5). The usage of these was investigated by utilizing EBV-transformed lymphoblastoid RNA from males affected with X-linked recessive HPT and normal males for RT-PCR analysis (3). These analyses in both the affected and normal individuals revealed that 2 upstream exons were spliced to the invariant exon 2 of SNTG2, but DNA sequence analysis revealed that the resultant transcripts did not have open reading frames (data not shown) and that they were therefore not likely to be of functional significance. These results were confirmed by analyzing alternative $S N T G 2$ transcripts that were obtained by use of 5 ' rapid amplification of cDNA ends (RACE) and human kidney Marathon-Ready cDNA (BD Biosciences) (30) (data not shown). The 23- to $25-\mathrm{kb}$ sequence deleted from Xq27.1 deleted $23-25 \mathrm{~kb}$ sequence is not known to contain any genes or ESTs, but nucleic acid identification X (NIX) analysis indicated the presence of 4 putative exons. Oligonucleotide primers specific for these were designed and utilized in RT-PCR analysis that used 35 cycles to facilitate detection of low levels of illegitimate transcripts (3) in RNA from EBV-transformed lymphoblastoids of a normal male, as normal human adult or embryonic parathyroid tissue was not available. The RT-PCR analysis failed to detect transcripts, thereby indicating that this 23 - to $25-\mathrm{kb}$ sequence deleted from $\mathrm{Xq} 27.1$ is likely to be a noncoding region, although it is important to note that illegitimate transcripts of developmental genes may occasionally not be detected in RNA from EBV-transformed lymphoblastoids $(3,17)$.

Sox 3 expression in developing parathyroids. The noncoding region encompassed by the 23 - to $25-\mathrm{kb}$ deleted sequence is situated 67 $\mathrm{kb}$ downstream of $\mathrm{SOX} 3$, and this location suggested the possibility that this region could regulate $S O X 3$ transcription. Indeed, 


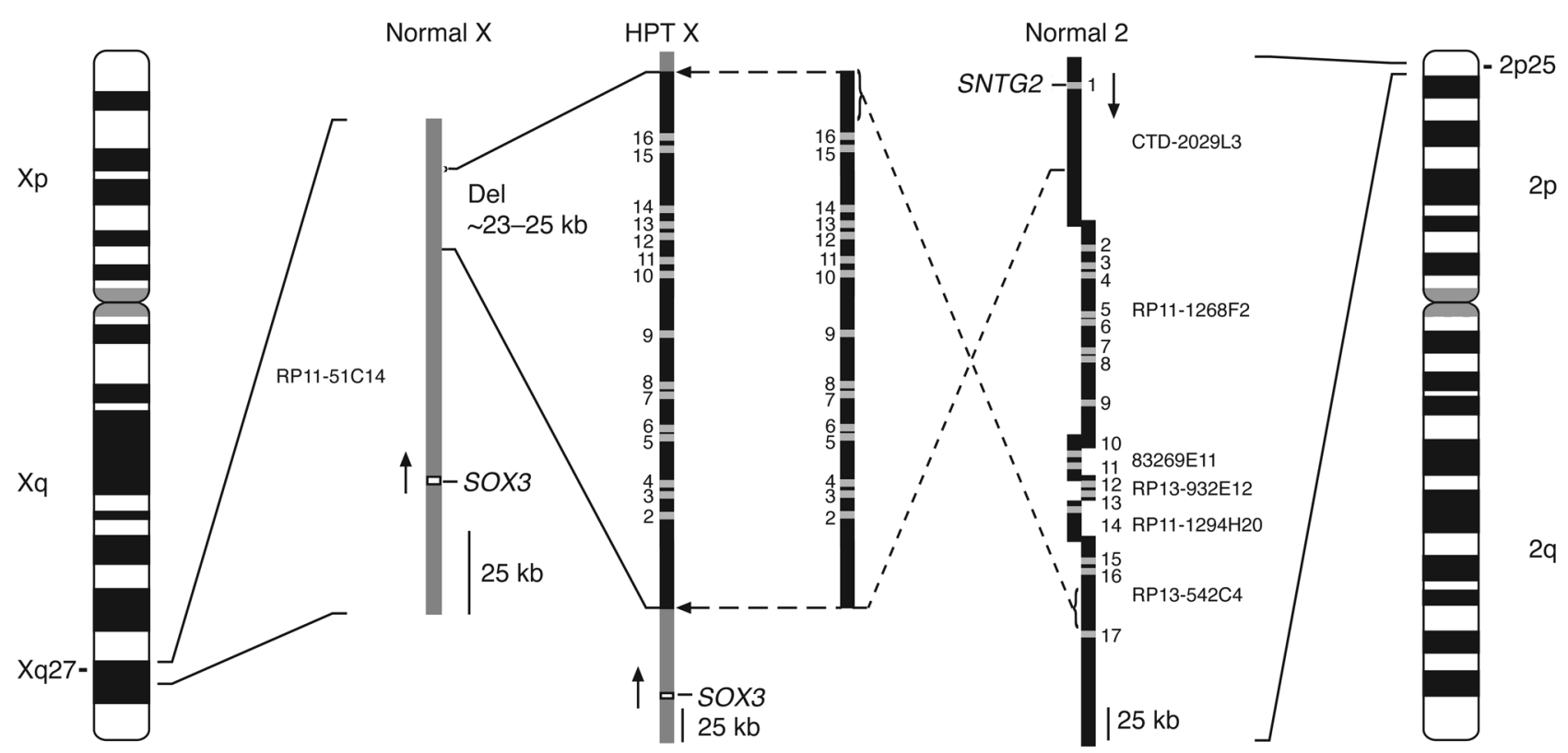

Figure 5

Molecular mechanism underlying the deletion-insertion abnormality involving chromosomes Xq27 and 2p25 in X-linked recessive HPT. The relationships of the deletion-insertion to that of BACs (Figures 1 and 4) from chromosomes 2p25 (CTD-2029L3, RP11-1268F2, XXfos-83269E11, RP13-932E12, RP11-1294H20, and RP13-542C4) and Xq27 (RP11-51C14) are shown. DNA sequence database analyses together with a characterization of the DNA sequences of the breakpoints (Figures 2 and 3 ) in X-linked recessive HPT patients established that the deletion within Xq27.1 extends approximately $23-25 \mathrm{~kb}$ and that the insertion from chromosome $2 \mathrm{p} 25.3$, which inverted upon insertion, extends approximately $305-340 \mathrm{~kb}$ and includes exons 2-16 of the SNTG2 gene. The location of this deletion-insertion is approximately $67 \mathrm{~kb}$ downstream of the gene encoding SOX3, which belongs to a family of transcription factors that are involved in vertebrate embryonic development (13). For example, abnormalities of SOX3 expression are associated with developmental anomalies involving the CNS, craniofacial bones, and pituitary (15-18, 41), while abnormalities of $S R Y$ expression are associated with disorders of sexual development (49). It is likely that the deletion-insertion causes a disruption of SOX3 regulatory elements and thereby exerts a position effect on SOX3 expression.

genomic elements such as enhancers, repressors, or insulators that may be involved in transcription regulation have been reported to be located up to $1 \mathrm{Mb}$ upstream or downstream of the transcriptional unit (14). As a first step in exploring such a possibility, we examined for Sox3 expression by in situ hybridization (Figure 6) in the pharyngeal pouches and developing parathyroids of mouse embryos, as such human tissues are not available. We also assessed for Casr expression, which has been reported to be particularly expressed in parathyroid and thyroid $\mathrm{C}$ cells that produce calcitonin (6), as a control. These studies revealed Casr expression, as early as 10.5 days post coitum (dpc), in the parathyroid precursor tissue at the endodermal margin of the third pharyngeal pouch (Figure 6). At this stage, on serial sections, Sox 3 expression was detected in an adjacent but nonoverlapping domain, in the posterior margin of the third, and also second, pharyngeal pouch. Sox3 expression was also observed at 13.5 and $15.5 \mathrm{dpc}$, in the developing parathyroids and thymic and thyroid rudiments, but this was dramatically reduced by $18.5 \mathrm{dpc}$. In contrast, Casr was strongly and uniformly expressed, at $18.5 \mathrm{dpc}$, in the parathyroids, while in the thyroid its expression was clustered in foci that may likely represent $C$ cells (6). Thus, Sox 3 is expressed between 10.5 and $15.5 \mathrm{dpc}$ in the second and third pharyngeal pouches and the developing parathyroids. These findings are consistent with the hypothesis that SOX3 misexpression may lead to abnormalities of parathyroid development. Such misexpression of SOX3 could be caused by a position effect resulting from the deletion-insertion $[\operatorname{del}(\mathrm{X})(\mathrm{q} 27.1)$ inv ins $(\mathrm{X} ; 2)(\mathrm{q} 27.1 ; \mathrm{p} 25.3)]$, which is situated $67 \mathrm{~kb}$ downstream of SOX3 and is associated with $\mathrm{X}$-linked recessive HPT.

\section{Discussion}

Our studies have identified a novel molecular deletion-insertion involving chromosomes Xq27.1 and 2p25.3 in patients with $\mathrm{X}$-linked recessive HPT due to parathyroid agenesis (11). Deletioninsertions and translocations are well documented in the etiology of cancers, e.g., thyroid carcinomas and leukemias $(31,32)$, and in nonfamilial forms of diseases such as DiGeorge and HDR syndromes $(1,2,12)$. However, to our knowledge, this is the first report of a stably inherited germline $\mathrm{X}$-autosomal rearrangement that causes disease. This deletion-insertion is likely to have arisen, in the founder, by nonhomologous recombination, and the (at) dinucleotide-rich repetitive sequence associated with the centromeric breakpoint is consistent with this postulated mechanism (33). This event appears to be an extraordinarily rare one that likely occurred once and more than a century ago in North America, as only 2 related kindreds with X-linked recessive HPT have been reported, and both are from Missouri $(4,5,10)$. Moreover, our identification of this deletion-insertion highlights the important role for genetic abnormalities that involve noncoding regions in causing disease, a feature that is likely to be of significance in the search for the molecular basis of other Mendelian inherited diseases for which coding region abnormalities have not been identified (14).

The manner in which this deletion-insertion $[\operatorname{del}(X)(q 27.1)$ inv ins $(\mathrm{X} ; 2)(\mathrm{q} 27.1 ; \mathrm{p} 25.3)]$, which involves a loss of $23-25 \mathrm{~kb}$ of non- 

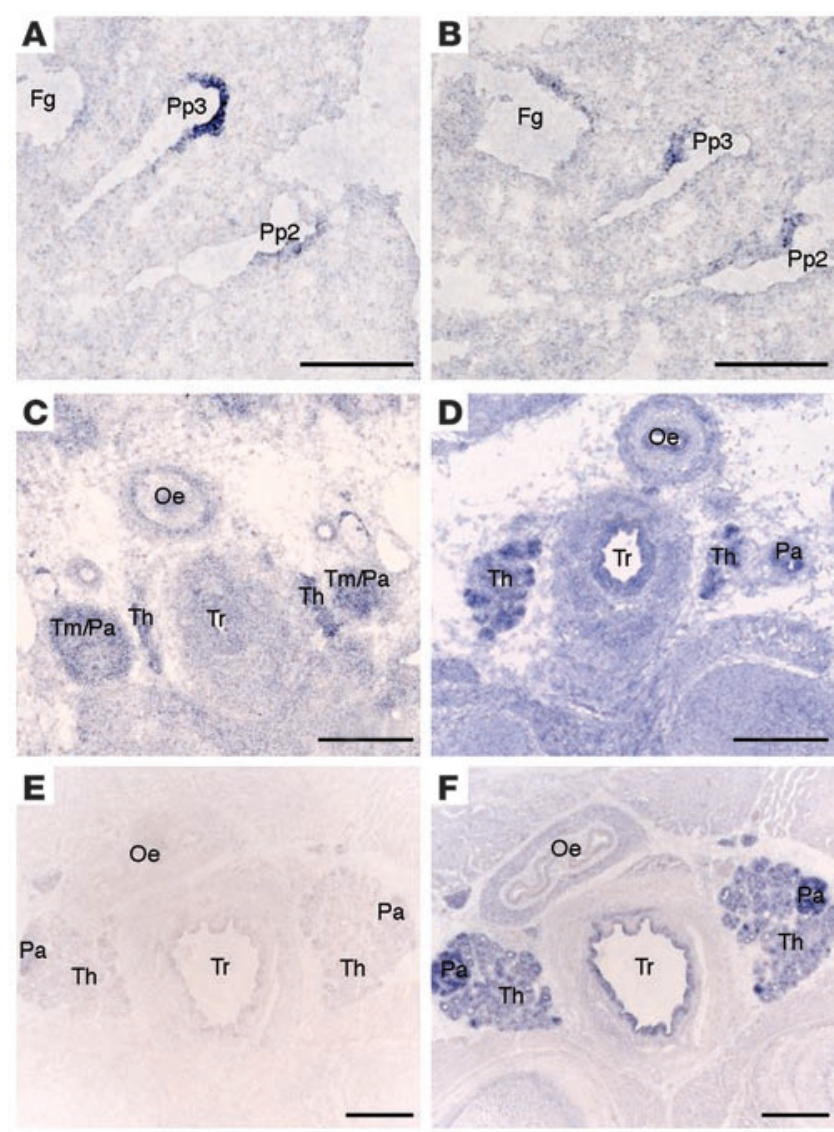

coding Xq27.1 sequence and an inverted insertion of $305-340 \mathrm{~kb}$ from chromosome 2 p25.3 to Xq27.1 in the founder (Figure 5), affects the embryonic development of the parathyroids from the third and fourth pharyngeal pouches in humans remains to be elucidated. At least 4 possible explanations could be considered. First is the possibility that trisomy of the inserted 2 p25.3 sequence may result in a dosage effect that alters parathyroid development. However, this seems unlikely, as patients with $2 \mathrm{p}$ trisomy have not been reported to suffer from hypocalcemia or HPT (34). Moreover, such 2p25.3 trisomy occurs in the X-linked recessive HPT carrier females, who are normocalcemic and do not have HPT $(4,5)$. Thus, increased dosage of $2 \mathrm{p} 25.3$ is not the cause of X-linked recessive HPT.

Second is the possibility that expression of abnormal splice variants of SNTG2 may disrupt parathyroid development. SNTG2 belongs to the family of SYNTROPHIN proteins, which contain at least one pleckstrin homology (PH) domain, a PSD-95, discslarge, Z0-1 (PDZ) domain, and a C-terminal syntrophin unique (SU) region (35). All of the syntrophin proteins associate with dystrophin and dystrobrevin through an interaction between the SU and adjacent PH domain $(29,36)$, while the PDZ domain interacts with receptors, ion channels, and other signaling proteins (37-39). Thus, an SNTG2 isoform may regulate parathyroid development by its action as an adaptor protein that either links transmembrane receptors to intracellular signaling cascades or facilitates recycling of $G$ protein-coupled receptors in the process of receptor resensitization. This possibility, although attractive, is unlikely, as the SNTG2 transcripts that result from the inser-

\section{Figure 6}

Expression of Sox3 and Casr in the developing parathyroids of mouse embryos. Transverse sections through the regions of the pharyngeal pouches and neck, from wild-type (normal) mouse embryos between 10.5 and $18.5 \mathrm{dpc}$, were hybridized with either Casr or Sox3 riboprobes. Casr, which is known to be expressed in the parathyroids and thyroids (6), was used as a control. (A) Casr expression was observed in the third pharyngeal pouch (Pp3) at 10.5 dpc. (B) On a serial section, Sox3 expression was observed in the posterior margins of the second (Pp2) and third pharyngeal pouch endoderm and also the foregut endoderm (Fg). (C) Sox3 and Casr (data not shown) expression were detected in the thyroid (Th) and in the thymic (Tm) and parathyroid $(\mathrm{Pa})$ rudiments at $13.5 \mathrm{dpc}$. (D) Sox3 expression continued in the developing thyroid and parathyroids at $15.5 \mathrm{dpc}$. (E) However, Sox3 expression had almost ceased in the developing thyroid and parathyroids by $18.5 \mathrm{dpc}$. (F) In contrast, Casr was seen to be strongly expressed in the developing thyroid and parathyroids on a serial section at $18.5 \mathrm{dpc}$. Oe, esophagus; Tr, trachea. These results demonstrate that Sox 3 is expressed, between $10.5 \mathrm{dpc}$ and $15.5 \mathrm{dpc}$, in the pharyngeal pouches and developing parathyroids of the mouse embryo. Scale bars, $0.25 \mathrm{~mm}$.

tion, which contains approximately $61 \mathrm{~kb}$ of intron 1 together with exons 2-16 (Figure 5), do not have open reading frames and hence lack the critical PDZ, SU, and PH domains.

Third is the possibility that the $23-25 \mathrm{~kb}$ deleted Xq27.1 sequence contains an unidentified gene whose loss disrupts parathyroid development. However, this is unlikely, as the $23-25 \mathrm{~kb}$ DNA sequence is not reported to contain genes or ESTs, and our studies using EBV-transformed lymphoblastoid RNA failed to detect any transcripts. Furthermore, a patient who has a deletion spanning the approximately $1-\mathrm{Mb}$ interval from FACTOR 9 (F9) to DXS984 (Figure 1) has been reported to suffer from hemophilia B and mental retardation but not hypocalcemia or HPT (13). Thus, it seems likely that this $23-25 \mathrm{~kb}$ deleted region is noncoding.

Fourth is the possibility that this noncoding region could contain regulatory regions for $S O X 3$, which is located $67 \mathrm{~kb}$ telomeric to the deletion-insertion. Thus, a disruption of such putative regulatory sequences that may alter SOX3 expression would be postulated to lead to parathyroid agenesis and X-linked recessive HPT. Our demonstration that Sox3 is expressed in the third pharyngeal pouch and developing parathyroids of mouse embryos between 10.5 and $15.5 \mathrm{dpc}$ (Figure 6) provides support for such a role of SOX3 in parathyroid genesis. SOX3 has not previously been shown to be expressed in the parathyroids, and thus our results add to the growing list of transcription factors, which include GCMB, GATA3, TBX1, HOXA3, PAX1, and PAX9, that operate in parathyroid development $(1,12,24,40)$. Sox3 is also expressed in the infundibulum of the developing pituitary and presumptive hypothalamus of mouse embryos, with its expression in the latter being maintained in the adult $(15,41)$. Moreover, SOX3 abnormalities are associated with X-linked hypopituitarism and with $\mathrm{X}$-linked mental retardation that occurs with growth hormone deficiency $(15,17,18)$.

SOX3 belongs to a family of genes encoding high-mobility group box transcription factors and is related to SRY, the sex-determining gene on the $\mathrm{Y}$ chromosome. The mouse homolog is expressed in the prestreak embryo and subsequently in the developing CNS that includes the region of the ventral diencephalon, which induces development of the anterior pituitary and gives rise to the hypothalamus $(15,41,42)$; olfactory placodes; and parathy- 
roids (Figure 6). Patients with X-linked hypopituitarism have been reported to have duplications involving a $686-\mathrm{kb}$ to $13-\mathrm{Mb}$ region that contains SOX3 (15-17), and overexpression of SOX3 has been reported to inhibit Wnt signaling, which has an important role in pituitary development (43). Furthermore, increased levels of SOX3 have been shown to cause developmental hypoplasia of tissues, such as the lens and otic placodes in fish embryos (44). Reduced levels of SOX3 expression also result in hypopituitarism. Thus, SOX3-null mice have abnormal pituitary development associated with hypopituitarism, craniofacial abnormalities, and midline CNS defects (41). These phenotypic features are similar to those observed in patients with X-linked hypopituitarism and with X-linked mental retardation and growth hormone deficiency who have in-frame duplications of $21 \mathrm{bp}$ or $33 \mathrm{bp}$ encoding for 7 or 11 alanines, respectively, in a polyalanine tract of the SOX3 gene $(17,18)$. The polyalanine tract expansion resulted in a reduction in SOX3 transcriptional activity that was associated with an impaired nuclear localization of the mutant protein (17). These findings demonstrate that pituitary development is sensitive to SOX3 dosage, and that both loss- and gain-of-function mutations can result in X-linked hypopituitarism $(15,16,18,41,45)$.

Patients with X-linked hypopituitarism have not been reported to suffer from HPT (15-18), and, conversely, the patients affected with X-linked recessive HPT do not suffer from hypopituitarism $(4,5,8)$. These clinical differences may be due to the differences in the temporal expression patterns of $S_{O X} 3$ in the pituitary and parathyroids or to interactions with different tissue-specific enhancers or repressors. Alternatively, they may be due to differences in the locations of the associated SOX3 genomic abnormalities, and it is important to note that X-linked hypopituitarism is associated with either duplications of the entire SOX3 coding region or with an intragenic expansion of a polyalanine tract (15-18), whereas X-linked recessive HPT is associated with a deletion that is approximately $67 \mathrm{~kb}$ downstream from the SOX3 coding region (Figure 5). This situation may be analogous to that reported to occur in disorders associated with abnormalities of the gene encoding sonic hedgehog ( $\mathrm{SHH})(14)$. $\mathrm{SHH}$ is a secreted protein that provides key inductive signals for the patterning of ventral neural tube, the anterior posterior limb axis, and the ventral somites (14). SHH gene abnormalities lead to holoprosencephaly type 3 (HPE3) and preaxial polydactyly (PPD) in humans (14). HPE3 is caused by deletions or point mutations that involve the coding region and result in haploinsufficiency of SHH, while PPD is caused by breakpoints or point mutations within a limb regulatory element that is 1 $\mathrm{Mb}$ upstream of SHH (14). These findings illustrate that phenotypes caused by mutations in regulatory elements can be very different from those caused by mutations of coding regions and that point mutations in regulatory elements at a distance as far as $1 \mathrm{Mb}$ from the gene promoter can have a detrimental affect on embryonic development (14). Thus, it seems likely that the differences between X-linked recessive HPT and hypopituitarism occur because the HPT deletion-insertion, which is approximately $67 \mathrm{~kb}$ downstream of $S O X 3$, involves disruption of regulatory elements, whereas the abnormalities resulting in hypopituitarism involve alterations of the SOX3 coding region. It is also important to note that the disruption in the regulatory region could also lead to upregulation or misregulation of the gene, thereby resulting in phenotypes due to gain-of-function (i.e., hypermorphic or neomorphic).
The location of the deletion-insertion approximately $67 \mathrm{~kb}$ downstream of SOX3 in X-linked recessive HPT patients is likely to result in altered SOX3 expression, as SOX3 expression has been reported to be sensitive to position effects caused by $\mathrm{X}$ chromosome abnormalities $(14,19)$. Indeed, reporter-construct studies of the mouse Sox 3 gene have demonstrated the presence of both $5^{\prime}$ and $3^{\prime}$ regulatory elements (46), and thus it is possible that the deletion-insertion in the X-linked recessive HPT patients may have a position effect on SOX3 expression and parathyroid development from the pharyngeal pouches. Indeed such position effects on SOX genes, which may be exerted over large distances, have been reported. For example, the very closely related Sox2 gene has been shown to have regulatory regions spread over a long distance, both $5^{\prime}$ and $3^{\prime}$ to the coding region (47), and disruption of sequences at some distance $3^{\prime}$ (>20 kb) have recently been reported to lead to loss of expression in the developing inner ear and absence of sensory cells, whereas expression in other sites is unaffected (48). Similarly for the SRY gene, which probably originated from SOX3 (13), both $5^{\prime}$ and $3^{\prime}$ deletions result in abnormalities of sexual development $(14,19,49)$, and translocation breakpoints more than $1 \mathrm{Mb}$ upstream of the SOX 9 gene have been reported to result in Campomelic dysplasia due to removal of elements that regulate SOX9 expression $(14,19)$. Thus, our studies, which have identified a molecular deletioninsertion that may cause a position effect on SOX3 expression leading to X-linked recessive HPT, point to a potential role for the SOX 3 gene in the embryological development of the parathyroid glands from the pharyngeal pouches.

\section{Methods}

Families. Two related kindreds from Missouri, USA, in whom idiopathic HPT had been inherited in an X-linked recessive manner in 5 or more generations $(4,5)$ and in whom linkage between the disease and polymorphic loci from chromosome Xq26-27 (Figure 1) had been established (7-9), were investigated. Venous blood was collected, with approved Human Studies Committee (Washington University School of Medicine) guidelines used to obtain informed consent, from 61 family members ( 6 affected males, 11 carrier females and 44 unaffected [ 16 males, 28 females]) and 9 unrelated males with idiopathic HPT, as previously reported $(7,8)$.

Analysis of DNA and RNA. DNA was prepared from peripheral blood leukocytes or from EBV-transformed lymphoblastoids, and RNA was prepared from EBV-transformed lymphoblastoids, as previously described (3). Oligonucleotide primers were designed for use in PCR amplification of DNA sequences, as previously reported $(3,9,20)$ to: detect SNPs; characterize the boundaries of deletions (ISP3 5'-GGGCTAACGAGAACGCAGAGGC-3'; XSPF 5'-GCTAGTAATGTTCTCTGTCTTGAATTTGCCC-3'; XSPR 5'-GGATCCTCATATCCCACTGTGGTCAAAGG-3') and insertions by use of STSs (STS a forward (F) 5'-AGGTGGCAATTTCAAGAGTGC-3', reverse (R) 5'-CCGTGTGAAGCCTATGACTGC-3'; STS b F 5'-GCAACTAAAGAAGGGCTTAGAGG-3', R 5'-CACAATACTTCTGGAGGCAAGG-3'; STS c F 5'-CCTAACTTTGCCCCTTCACTGC-3', R 5'-TGGAGGAAGAAAACTTTGAGC-3'; STS d F 5'-TGCCTCAGTCTCCTCACTTGGC-3', R 5'-CTCATATCCTGCCACCAAATTCC-3'; STS e F 5'-TGGCTCACACCTGTAAATACC-3', R 5'GCAACATCGGTGGGAACAGCAG-3'); generate probes for screening of YAC, PAC, and Fosmid libraries; determine the chromosomal locations of novel genomic sequences by use of a rodent-human monochromosomal somatic cell hybrid panel (ISP1 5'-CCCATTTAAGTATAAAACTTATGTTCCTCC-3'; ISP2 5'-CGTGAATCCCTGCATTCCCGTGAGG-3'); and identify cDNAs using 5' RACE (30). Oligonucleotide primers were 
also used with RNA in RT-PCR that utilized avian myeloblastosis virus reverse transcriptase (Life Sciences Inc.) to identify transcripts, as previously described $(3,9)$. The DNA sequences of PCR products were determined using Taq polymerase cycle sequencing and a semi-automated laser detection system (Sequencer 373A; Applied Biosystems), as previously reported (9). High-molecular-weight DNA was used for restriction mapping using 4-fold excess of the "rare cutter" endonuclease SfiI and the resulting fragments separated by PFGE; transferred by the method of Southern to Hybond- $\mathrm{N}^{+}$(Amersham Biosciences UK Ltd.) membranes, which were hybridized using a $\left.{ }^{32} \mathrm{P}\right]$-labeled DNA probe for SOX3 (F 5'TCCGAGCAGGTATATAAGGGG-3'; R 5'-TTCGCACTACTCTTGCCTGC-3'); and visualized by autoradiography (20).

Isolation, preparation, and analysis of YAC, BAC, PAC, and Fosmid clones. BAC clones were identified using BLAST and the NCBI GenBank database. YAC clones were identified from the CEPH megaYAC library (27), and PAC and Fosmid clones were identified from the human chromosome 2-specific filter libraries LL02NP04 and LL02NC03 (26), respectively, using [ $\left.{ }^{32} \mathrm{P}\right]$-labeled DNA probes. These DNA probes were generated from PCR products using the Megaprime DNA labeling system, according to the manufacturer's recommendations (Amersham Biosciences UK Ltd.). The DNA sequences of the YAC, PAC, and Fosmid clones were not available, and the terminal portions of these clones were isolated using ligation-mediated end rescue methods such as inverse or vectorette PCR $(20,22,50)$ and the DNA sequences of the products determined. A contig consisting of these clones was assembled, as described previously (20). These clones were prepared and labeled by incorporation of labeled dUTP for use on metaphase spreads and FISH.

Metaphase- and DNA fiber-FISH. Metaphase chromosome spreads were prepared from EBV-transformed lymphoblastoid cells (3) following standard procedures, and single- and dual-color FISH was performed, as previously described (28), using biotin- and digoxigenin-labeled probes. Biotinylated probes were detected with Texas red-conjugated streptavidin (Invitrogen Corp.), and digoxigenin labeled probes were detected with a mouse anti-digoxigenin antibody (Roche Diagnostics Corp.) and goat anti-mouse Alexa-488 (Invitrogen Corp.). The slides were mounted with VECTASHIELD (Vector Laboratories) containing DAPI for chromosome counterstaining. A minimum of 25 metaphases were analyzed for each hybridization experiment using an Olympus BX-51 epifluorescence microscope coupled to a SenSys charge-coupled device camera (Photometrics; Roper Scientific Inc.). Texas red, Alexa-488, and DAPI fluorescence images were taken as separate gray-scale images using specific filter combinations and then pseudocolored and merged using the software package Genus version 2.81 (Applied Imaging International). DNA fiber slides were prepared as described previously (28) and triple-color FISH performed using biotin-, digoxigenin-, and Alexa-594-labeled probes. Biotinylated probes were visualized using fluorescein-conjugated streptavidin, and digoxigenin-labeled probes were visualized using mouse anti-digoxigenin (Roche Diagnostics Corp.) followed by a layer of rabbit anti-mouse Cy5 (Stratech Scientific Ltd.) and a final layer of goat anti-rabbit Cy5 (Stratech Scientific Ltd.). Probes labeled with Alexa-594 dUTP (Invitrogen Corp.) were visualized directly. The slides were mounted with VECTASHIELD (Vector Laboratories), and images were captured and analyzed using a Bio-Rad MRC1024 confocal microscope (Bio-Rad Laboratories).

Genomic library construction. Leukocyte DNA from a male affected with $\mathrm{X}$-linked recessive HPT (individual IV.15 from family W/81; Figure 2) and an unaffected (control) male (individual IV.14 from family W/81; Figure 2) was digested with BamHI, EcoRI, HindIII, HpaII, and RsaI and used to construct genomic libraries utilizing the Vectorette II system (Sigma-Aldrich) (22). In order to enrich the source of the HPT insert abnormality, flow-sorted X chromosomes extracted from EBV-trans- formed lymphoblastoids (51) of a male affected with X-linked recessive HPT (individual IV.15 from family W/81; Figure 2) were utilized to generate EcoRI, RsaI, and TaqI vectorette libraries, using previously reported methods $(22,51)$. These libraries were employed as template DNA for PCR amplification using XSPs in conjunction with a Vectorette unit binding primer (Sigma-Aldrich). Targeted PCR products were identified using an oligomer-extension "hot blot" assay, as previously described (23). Targeted products were then excised from a duplicate gel, the DNA extracted, and their sequence determined using Taq polymerase cycle sequencing $(9,12,24)$.

Detection of Sox 3 and Casr expression in mouse embryos by in situ bybridization. Parkes mice were staged according to the date of the vaginal plug. Embryos were harvested, fixed overnight in $4 \%$ paraformaldehyde at $4{ }^{\circ} \mathrm{C}$, cryoprotected in $20 \%$ sucrose, and embedded in OCT (BDH), using previously described methods (41). In situ hybridizations using cryosections were performed as described previously (52) using Sox3 (41) and Casr riboprobes. The Casr riboprobe, which was $979 \mathrm{bp}$ in size, was generated by RT-PCR using total RNA from mouse (129/Ola) embryonic stem cells together with primers specific to exon 2 (5'-AGACCAGAGTCTGTGGAGTGC-3', nucleotides 685-705; NM_013803 Mus musculus Calciumsensing receptor [Casr], mRNA) and exon 4 (5'-CCTTCCTCGTGACTTCTCACG-3', nucleotides 1,663-1,643). The RT-PCR product was subcloned into pGEM-T, the construct linearized using NdeI, and an antisense digoxigenin-labeled Casr probe generated using T7 RNA polymerase. All experiments on mice were performed using protocols approved under the United Kingdom Animal (Scientific Procedures) Act. All animal procedures were authorized by the United Kingdom Home Office.

Database searches. Nucleotide sequence analyses and comparisons were performed using the GenBank/BLAST (http://www.ncbi.nlm.nih.gov/ BLAST/), Ensembl/BLAST (http://www.ensembl.org), and NIX (no longer available) databases and programs available in 2001-2004. OMIM was accessed on the World Wide Web (http://www.ncbi.nlm.nih.gov/omim/).

Linkage analysis. Data from the present and previously reported studies $(7,8)$ of the 2 related kindreds, families $\mathrm{P} / 60$ (4) and W/81 (5), were pooled for linkage studies. Conventional 2-point LOD scores were calculated using a web-based linkage computer application, GLUE (LITBIO; http://www.litbio.org).

\section{Acknowledgments}

This work was supported by grants from the MRC (United Kingdom) (to M.R. Bowl, M.A. Nesbit, B. Harding, K. Rizzoti, R. Lovell-Badge, and R.V. Thakker), the Wellcome Trust (United Kingdom) (to E. Levy, A. Jefferson, E. Volpi, and R.V. Thakker), and the Shriners Hospitals for Children (USA) (to M.P. Whyte). M.R. Bowl was an MRC PhD Student. We thank K. Boone (Weatherall Institute of Molecular Medicine) for help with flowsorting of X chromosomes.

Received for publication December 12, 2004, and accepted in revised form July 12, 2005.

Address correspondence to: Rajesh V. Thakker, Academic Endocrine Unit, Nuffield Department of Medicine, University of Oxford, Oxford Centre for Diabetes, Endocrinology and Metabolism (OCDEM), Churchill Hospital, Headington, Oxford OX3 7LJ, United Kingdom. Phone: 44-1865-857501; Fax: 44-1865857502; E-mail: rajesh.thakker@ndm.ox.ac.uk.

Michael R. Bowl and M. Andrew Nesbit contributed equally to this work. 
1. Thakker, R.V. 2004. Genetics of endocrine and metabolic disorders: parathyroid. Rev. Endocr. Metab. Disord. 5:37-51.

2. Baldini, A. 2003. DiGeorge's syndrome: a gene at last. Lancet. 362:1342-1343.

3. Parkinson, D.B., and Thakker, R.V. 1992. A donor splice site mutation in the parathyroid hormone gene is associated with autosomal recessive hypoparathyroidism. Nat. Genet. 1:149-152.

4. Peden, V.H. 1960. True idiopathic hypoparathyroidism as a sex-linked recessive trait. Am. J. Hum. Genet. 12:323-337.

5. Whyte, M.P., and Weldon, V.V. 1981. Idiopathic hypoparathyroidism presenting with seizures during infancy: X-linked recessive inheritance in a large Missouri kindred. J. Pediatr. 99:608-611.

6. Brown, E.M., and MacLeod, R.J. 2001. Extracellular calcium sensing and extracellular calcium signaling. Physiol. Rev. 81:239-297.

7. Thakker, R.V., Davies, K.E., Whyte, M.P., Wooding, C., and O'Riordan, J.L. 1990. Mapping the gene causing X-linked recessive idiopathic hypoparathyroidism to Xq26-Xq27 by linkage studies. J. Clin. Invest. 86:40-45.

8. Trump, D., et al. 1998. Localisation of X linked recessive idiopathic hypoparathyroidism to a 1.5 $\mathrm{Mb}$ region on Xq26-q27. J. Med. Genet. 35:905-909.

9. Nesbit, M.A., et al. 2004. X-linked hypoparathyroidism region on $\mathrm{Xq} 27$ is evolutionarily conserved with regions on $3 q 26$ and $13 q 34$ and contains a novel P-type ATPase. Genomics. 84:1060-1070.

10. Mumm, S., Whyte, M.P., Thakker, R.V., Buetow, K.H., and Schlessinger, D. 1997. mtDNA analysis shows common ancestry in two kindreds with $\mathrm{X}$-linked recessive hypoparathyroidism and reveals a heteroplasmic silent mutation. Am. J. Hum. Genet. 60:153-159.

11. Whyte, M.P., Kim, G.S., and Kosanovich, M. 1986. Absence of parathyroid tissue in sex-linked recessive hypoparathyroidism [letter]. J. Pediatr. 109:915.

12. Van Esch, H., et al. 2000. GATA3 haplo-insufficiency causes human HDR syndrome. Nature. 406:419-422.

13. Stevanovic, M., Lovell-Badge, R., Collignon, J., and Goodfellow, P.N. 1993. SOX3 is an X-linked gene related to SRY. Hum. Mol. Genet. 2:2013-2018.

14. Kleinjan, D.A., and van Heyningen, V. 2005. Longrange control of gene expression: emerging mechanisms and disruption in disease. Am. J. Hum. Genet. 76:8-32.

15. Solomon, N.M., et al. 2004. Array comparative genomic hybridisation analysis of boys with $\mathrm{X}$ linked hypopituitarism identifies a $3.9 \mathrm{Mb}$ duplicated critical region at Xq27 containing SOX3. J. Med. Genet. 41:669-678.

16. Solomon, N.M., et al. 2002. Increased gene dosage at Xq26-q27 is associated with X-linked hypopituitarism. Genomics. 79:553-559.

17. Woods, K.S., et al. 2005. Over- and underdosage of SOX3 is associated with infundibular hypoplasia and hypopituitarism. Am. J. Hum. Genet. 76:833-849.

18. Laumonnier, F., et al. 2002. Transcription factor SOX3 is involved in X-linked mental retardation with growth hormone deficiency. Am. J. Hum.
Genet. 71:1450-1455.

19. Kleinjan, D.J., and van Heyningen, V. 1998. Position effect in human genetic disease. Hum. Mol. Genet. 7:1611-1618.

20. Trump, D., et al. 1996. Construction of a YAC contig and an STS map spanning 3.6 megabase pairs in Xp22.1. Hum. Genet. 97:60-68.

21. Hui, E.K., Wang, P.C., and Lo, S.J. 1998. Strategies for cloning unknown cellular flanking DNA sequences from foreign integrants. Cell. Mol. Life Sci. 54:1403-1411.

22. Proffitt, J., Fenton, J., Pratt, G., Yates, Z., and Morgan, G. 1999. Isolation and characterisation of recombination events involving immunoglobulin heavy chain switch regions in multiple myeloma using long distance vectorette PCR (LDV-PCR). Lenkemia. 13:1100-1107.

23. Parker, J.D., and Burmer, G.C. 1991. The oligomer extension "hot blot": a rapid alternative to Southern blots for analyzing polymerase chain reaction products. Biotechniques. 10:94-101.

24. Nesbit, M.A., et al. 2004. Characterization of GATA3 mutations in the hypoparathyroidism, deafness, and renal dysplasia (HDR) syndrome. J. Biol. Chem. 279:22624-22634.

25. Kelsell, D.P., et al. 1995. Development of a panel of monochromosomal somatic cell hybrids for rapid gene mapping. Ann. Hum. Genet. 59:233-241.

26. Gingrich, J.C., et al. 1996. Construction and characterization of human chromosome 2-specific cosmid, fosmid, and PAC clone libraries. Genomics. 32:65-74.

27. Chumakov, I., et al. 1992. Continuum of overlapping clones spanning the entire human chromosome 21q. Nature. 359:380-387.

28. Bochukova, E.G., Jefferson, A., Francis, M.J., and Monaco, A.P. 2003. Genomic studies of gene expression: regulation of the Wilson disease gene. Genomics. 81:531-542.

29. Piluso, G., et al. 2000. Gamma1- and gamma2-syntrophins, two novel dystrophin-binding proteins localized in neuronal cells. J. Biol. Chem. 275:15851-15860.

30. Frohman, M.A., Dush, M.K., and Martin, G.R. 1988. Rapid production of full-length cDNAs from rare transcripts: amplification using a single genespecific oligonucleotide primer. Proc. Natl. Acad. Sci. U. S. A. 85:8998-9002.

31. Grieco, M., et al. 1990. PTC is a novel rearranged form of the ret proto-oncogene and is frequently detected in vivo in human thyroid papillary carcinomas. Cell. 60:557-563.

32. Faderl, S., et al. 1999. The biology of chronic myeloid leukemia. N. Engl. J. Med. 341:164-172.

33. Edelmann, L., et al. 2001. AT-rich palindromes mediate the constitutional $\mathrm{t}(11 ; 22)$ translocation. Am. J. Hum. Genet. 68:1-13.

34. Aviram-Goldring, A., et al. 2000. Molecular cytogenetic studies in three patients with partial trisomy $2 \mathrm{p}$, including CGH from paraffin-embedded tissue. Am. J. Med. Genet. 91:74-82.

35. Albrecht, D.E., and Froehner, S.C. 2002. Syntrophins and dystrobrevins: defining the dystrophin scaffold at synapses. Neurosignals. 11:123-129.

36. Kachinsky, A.M., Froehner, S.C., and Milgram, S.L.
1999. A PDZ-containing scaffold related to the dystrophin complex at the basolateral membrane of epithelial cells. J. Cell Biol. 145:391-402.

37. Hogan, A., et al. 2001. Interaction of gamma 1-syntrophin with diacylglycerol kinase-zeta. Regulation of nuclear localization by PDZ interactions. J. Biol. Chem. 276:26526-26533.

38. Oak, S.A., Russo, K., Petrucci, T.C., and Jarrett, H.W. 2001. Mouse alpha1-syntrophin binding to Grb2: further evidence of a role for syntrophin in cell signaling. Biochemistry. 40:11270-11278.

39. Kornau, H.C., Schenker, L.T., Kennedy, M.B., and Seeburg, P.H. 1995. Domain interaction between NMDA receptor subunits and the postsynaptic density protein PSD-95. Science. 269:1737-1740.

40. Scambler, P.J. 2000. The 22q11 deletion syndromes. Hum. Mol. Genet. 9:2421-2426.

41. Rizzoti, K., et al. 2004. SOX3 is required during the formation of the hypothalamo-pituitary axis. Nat. Genet. 36:247-255.

42. Collignon, J., et al. 1996. A comparison of the properties of Sox-3 with Sry and two related genes, Sox-1 and Sox-2. Development. 122:509-520.

43. Zorn, A.M., et al. 1999. Regulation of Wnt signaling by Sox proteins: XSox 17 alpha/beta and XSox3 physically interact with beta-catenin. Mol. Cell. 4:487-498.

44. Koster, R.W., Kuhnlein, R.P., and Wittbrodt, J. 2000. Ectopic Sox3 activity elicits sensory placode formation. Mech. Dev. 95:175-187.

45. Schlosser, G., and Ahrens, K. 2004. Molecular anatomy of placode development in Xenopus laevis. Dev. Biol. 271:439-466.

46. Brunelli, S., Silva Casey, E., Bell, D., Harland, R., and Lovell-Badge, R. 2003. Expression of Sox3 throughout the developing central nervous system is dependent on the combined action of discrete, evolutionarily conserved regulatory elements. Genesis. 36:12-24.

47. Uchikawa, M., Ishida, Y., Takemoto, T., Kamachi, Y., and Kondoh, H. 2003. Functional analysis of chicken Sox 2 enhancers highlights an array of diverse regulatory elements that are conserved in mammals. Dev. Cell. 4:509-519.

48. Kiernan, A.E., et al. 2005. Sox 2 is required for sensory organ development in the mammalian inner ear. Nature. 434:1031-1035.

49. Berta, P., et al. 1990. Genetic evidence equating SRY and the testis-determining factor. Nature. 348:448-450.

50. Hui, E.K., Wang, P.C., and Lo, S.J. 1998. Strategies for cloning unknown cellular flanking DNA sequences from foreign integrants. Cell. Mol. Life Sci. 54:1403-1411.

51. Carter, N.P., et al. 1990. Study of X chromosome abnormality in XX males using bivariate flow karyotype analysis and flow sorted dot blots. Cytometry. 11:202-207.

52. Schaeren-Wiemers, N., and Gerfin-Moser, A. 1993. A single protocol to detect transcripts of various types and expression levels in neural tissue and cultured cells: in situ hybridization using digoxigenin-labelled cRNA probes. Histochemistry. 100:431-440. 